\title{
The Spectroscopy of ${ }^{183} \mathrm{Tl}$ : an Extreme Case of Prolate-Oblate Shape-Competition
}

\author{
W. Reviol*, L. L. Riedinger*, C. R. Bingham*, W. Weintraub*, D. Jenkins ${ }^{\dagger}$, R. Wadsworth ${ }^{\dagger}$, \\ A. N. Wilson ${ }^{\dagger}$, S. Juutinen ${ }^{\ddagger}$, K. Helariutta ${ }^{\ddagger}$, M. P. Carpenter ${ }^{\S}$, R. V. F. Janssens ${ }^{\diamond}$, \\ D. Seweryniak ${ }^{\diamond}$, J. Uusitalo ${ }^{\circ}$, I. Wiedenhöver ${ }^{\circ}$, C. J. Gross ${ }^{\prime l}$, K. S. Toth"l, J. C. Batchelder ${ }^{\pi}$, \\ J. A. Cizewski ${ }^{\S}$
}

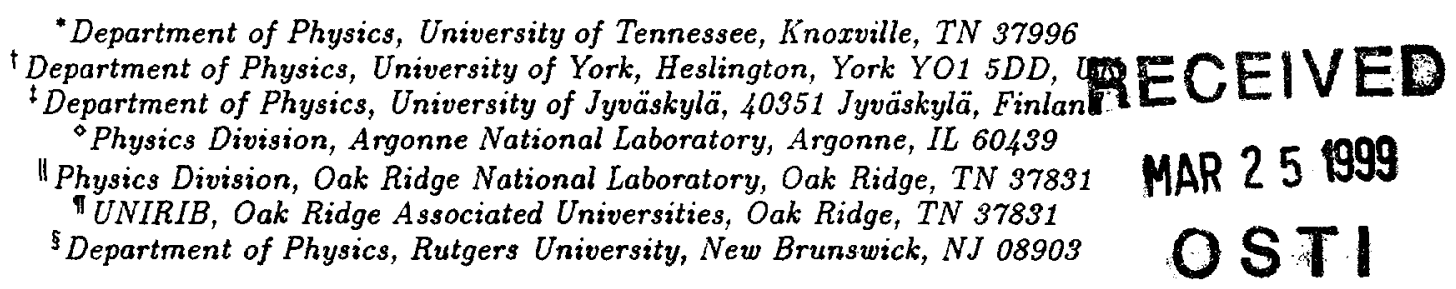

\begin{abstract}
The yrast sequence in ${ }^{183} \mathrm{Tl}$ has been studied for the first time in recoil-mass and decay tagged $\gamma$-ray spectroscopic measurements. A rotational-like cascade of seven transitions is observed down to the bandhead with spin $13 / 2^{+}$. In contrast to adjacent nuclei, links from the yrast band to a lower lying weakly deformed (oblate) structure are not observed. It appears that the prolate energy minimum in ${ }^{183} \mathrm{Tl}$ drops significantly compared to ${ }^{185} \mathrm{Tl}$ and minimizes below the neutron $i_{13 / 2}$ midshell $(N \leq 102)$. Possibilities for the decay out of the band in ${ }^{183} \mathrm{Tl}$ are discussed.
\end{abstract}

\section{INTRODUCTION}

The mercury-lead region, particularly at $A \leq 190$, has provided textbook examples for the shape coexistence phenomenon [1]. Shape transitions from weakly deformed $\left(\beta_{2} \sim 0.15\right)$ oblate $(\mathrm{Hg}$ nuclei) or spherical states ( $\mathrm{Pb}$ nuclei) to excited well deformed $\left(\beta_{2} \sim 0.25\right)$ prolate minima have been observed at very low spin (I > $2 \hbar$ ), see Refs. [1] and [2-4]. Coexistence between these oblate and prolate shapes is also seen in light Tl nuclei $(Z=81)$ [5]. The proton intruder orbitals such as the $i_{13 / 2}$ states are low- $K$ and downsloping as a function of deformation for $\mathrm{Tl}$ nuclei and thus are expected to stabilize the prolate minimum that is analogous to that found in adjacent $\mathrm{Hg}$ and $\mathrm{Pb}$ nuclei. Prolate bands built on $\pi i_{13 / 2}, \pi h_{9 / 2}$, and $\pi f_{7 / 2}$ orbitals have been observed in ${ }^{187} \mathrm{Tl}[6,7]$ and in ${ }^{185} \mathrm{Tl}[7]$. Whereas the prolate band in even-mass $\mathrm{Hg}$ and $\mathrm{Pb}$ nuclei minimizes in excitation energy at $N=103$ [8] or close to this neutron number [4], the prolate bands in Tl nuclei viz. the yrast bands based on the $i_{13 / 2}$ intruder are predicted [7] to drop further in the next lighter isotopes $(N=102$ and 100). This trend seems to highlight the shape-driving nature of the high-j proton intruders involved in the formation of the prolate minimum in the $\mathrm{Hg}, \mathrm{Tl}$, and $\mathrm{Pb}$ isotopes. Clearly, the exploration of the yrast structure of the $\mathrm{Tl}$ isotopes towards lower neutron numbers is of importance.

The results of a $\gamma$-ray spectroscopic study of the next lighter isotope, ${ }^{183} \mathrm{Tl}$, are discussed in the present paper. This nucleus and other very neutron-deficient nuclei in this mass region are difficult to study, since the fusion-evaporation cross sections are small compared to the fission yield, which typically accounts for $>90 \%$ of the decay of the compound system and introduces a large background. Therefore, to be sensitive for excited states of a particular evaporation residue, in-beam $\gamma$-ray spectroscopic methods need to be combined with mass separation of the recoiling nuclei or/and recoil decay tagging [9]. However, the strongest known line in the $\alpha$ decay of ${ }^{183} \mathrm{Tl}\left(E_{\alpha}\left(9 / 2^{-} \rightarrow 9 / 2^{-}\right)=6.38 \mathrm{MeV}\right)[10]$ represents a branch of $\sim 1.5 \%$ only. Thus, in the present case the RDT method is less suitable than e.g. for the neighboring $\mathrm{Pb}$ nuclei and the results presented hereafter 
are mainly based on the spectroscopy of ${ }^{183} \mathrm{Tl}$ with mass identification and identification of the atomic number by the $\mathrm{x}$-ray yield.

\section{EXPERIMENTAL TECHNIQUES AND RESULTS}

In a recent experiment with Gammasphere [11] at the Argonne Fragment Mass Analyzer (FMA) [12], the ${ }^{108} \mathrm{Pd}\left({ }^{78} \mathrm{Kr}, \mathrm{p} 2 \mathrm{n}\right)$ reaction at $340 \mathrm{MeV}$ was used to study ${ }^{183} \mathrm{Tl}$. Prompt $\gamma$ radiation from the target was detected with $93 \mathrm{Ge}$ counters of coaxial and four of planar (LEPS) type, all surrounded by BGo Compton suppressors. The FMA was used to separate the evaporation residues from fission products and primary beam. The mass/charge ratio of the evaporation residues was determined at the FMA focal plane using a positionsensitive parallel-grid avalanche counter (PGAC). These recoils were subsequently implanted in a double-sided Si strip detector (DSSD) located $40 \mathrm{~cm}$ downstream, which was used to detect the energies of recoils $\left(E_{r}\right)$ and decay particles $\left(E_{\alpha}\right)$ as well as their positions. The time-of-flight (TOF) of the recoils between GAMMASPHERE and PGAC and between PGAC and DSSD was also measured. A total of $\sim 10^{8}$ events were recorded under the trigger conditions, recoil- $\gamma^{n}(n \geq 1)$ or DSSD-decay.

The choice of a nearly symmetric reaction, which is in favor of a high FMA efficiency, causes a rather large amount of scattered beam at the FMA focal plane. In order to reduce the contamination from the scattered beam particles in the mass spectrum, a two-dimensional gate on the $E_{r}$ versus TOF (PGAC-DSSD) matrix is required for the data analysis. The resulting mass spectrum obtained for the PGAC X-position is shown in Fig. 1 (top). Peaks corresponding to $A=180$ to 184 recoils are observed for three charge states $(Q=30,31,32)$. The bottom part of Fig. 1 shows the $\gamma$-ray spectrum enhanced in $A=183$ by appropriate gating on the $\mathrm{x}$ - and $y$-position of recoils at the focal plane and on the TOF between GAMMASPHERE and the PGAC. Most of the strong $\gamma$-ray lines in this mass 183 gate can be identified as known transitions in ${ }^{183} \mathrm{Au}$ [13] and ${ }^{183} \mathrm{Hg}$ [14]. The remaining strong peaks in this mass-gated spectrum, labeled by their energies (in keV), are assigned to ${ }^{183} \mathrm{Tl}$. This assignment is based on $(i)$ the absence of concidence relationships between these new $\gamma$ rays and the known transitions in ${ }^{183} \mathrm{Au}$ or ${ }^{183} \mathrm{Hg}$, and (ii) coincident $\mathrm{Tl} \mathrm{x}$-rays, when gating on the 160-, 260-, 355-keV lines. It is further supported by statistical model calculations, which for the given reaction predict that the p2n channel leading to ${ }^{183} \mathrm{Tl}$ is stronger than the competing fusion-evaporation channels.

Fig. 2 shows $\gamma$-ray coincidence spectra attributed to ${ }^{183} \mathrm{Tl}$, obtained from the mass-gated $E_{\gamma}-E_{\gamma}$ matrix (unpacked $\gamma-\gamma-\gamma$ and higher fold events). All peaks labeled are in concidence with each other. The top spectrum shows the sum of gates placed on the $160-\mathrm{keV}$ to $514-\mathrm{keV}$ transitions. At the bottom, the spectrum gated by the $160-\mathrm{keV} \gamma$ ray is shown for comparison. Striking features of these spectra are (i) the regular energy spacings of the peaks, (ii) the absence of additional peaks that would disturb this spacing, and (iii) an intensity pattern that allows us to arrange these $\gamma$ rays in a cascade with increasing $E_{\gamma}$ from the bottom to the top of the sequence (see also next section). Obviously, the spectra of Fig. 2 display the yrast sequence in ${ }^{183} \mathrm{Tl}$, which exhibits a rotational behavior.

Spectroscopy of the ${ }^{182,183} \mathrm{Tl}$ nuclei has been also a byproduct of a study of ${ }^{182} \mathrm{~Pb}$ at RITU (Jyväskylä) [15], where a similar experimental setup and a ${ }^{42} \mathrm{Ca}$ induced reaction was used. At RITU, a $6.40 \mathrm{MeV} \alpha$ line $\left({ }^{183 \mathrm{~m}} \mathrm{Tl}, t_{1 / 2}=35 \mathrm{~ms}\right)$ was found [15], thought to be unobserved in the $\alpha$ spectra of Refs. $[10,16]$ and tentatively attributed to ${ }^{183} \mathrm{Tl}$, however, with some ambiguity on the mass assignment. The present data confirm this $\alpha$ line and allow for a firm assignment of it to ${ }^{183} \mathrm{Tl}$ [15]. The $\gamma$-ray spectrum correlated with this $\alpha$ line obtained at RITU [15] and a preliminary RDT analysis of the present data is consistent with the proposed assignment of the $\gamma$ rays in Fig. 2 to ${ }^{183} \mathrm{Tl}$. Furthermore, there is some indication for a weak $421 \mathrm{keV} \gamma$ ray in both the RDT and mass-gated spectra (not seen in Fig. 2), being the best candidate for the expected $11 / 2^{-} \rightarrow 9 / 2^{-}$transition ${ }^{183} \mathrm{Tl}$ which is seen in the heavier odd-mass isotopes and associated there with an oblate shape. However, the association of this $\gamma$ ray with ${ }^{183} \mathrm{Tl}$ should be viewed as a tentative assignment.

\section{DISCUSSION}

While the $260-, 355-, 439-, 514-, 581-\mathrm{keV}$ sequence in ${ }^{183} \mathrm{Tl}$ resembles the well-deformed yrast bands in the heavier $\mathrm{Tl}$ isotopes and neighboring $\mathrm{Hg}$ and $\mathrm{Pb}$ isotopes, the bottom part of this band and its decay-out are significantly different from those cases in that several strong transitions other than the 160 -keV line are absent in the spectra. This difference is probably best seen in the spectrum gated by the $160-\mathrm{keV}$ line (Fig. 2, bottom). If the $160-\mathrm{keV}$ line in ${ }^{183} \mathrm{Tl}$ were the analog to the $207-\mathrm{keV} 17 / 2^{+} \rightarrow 13 / 2^{+}$"decay-out" transition in ${ }^{185} \mathrm{Tl}$ (see 


\section{DISCLAIMER}

Portions of this document may be illegible in electronic image products. Images are produced from the best available original document. 

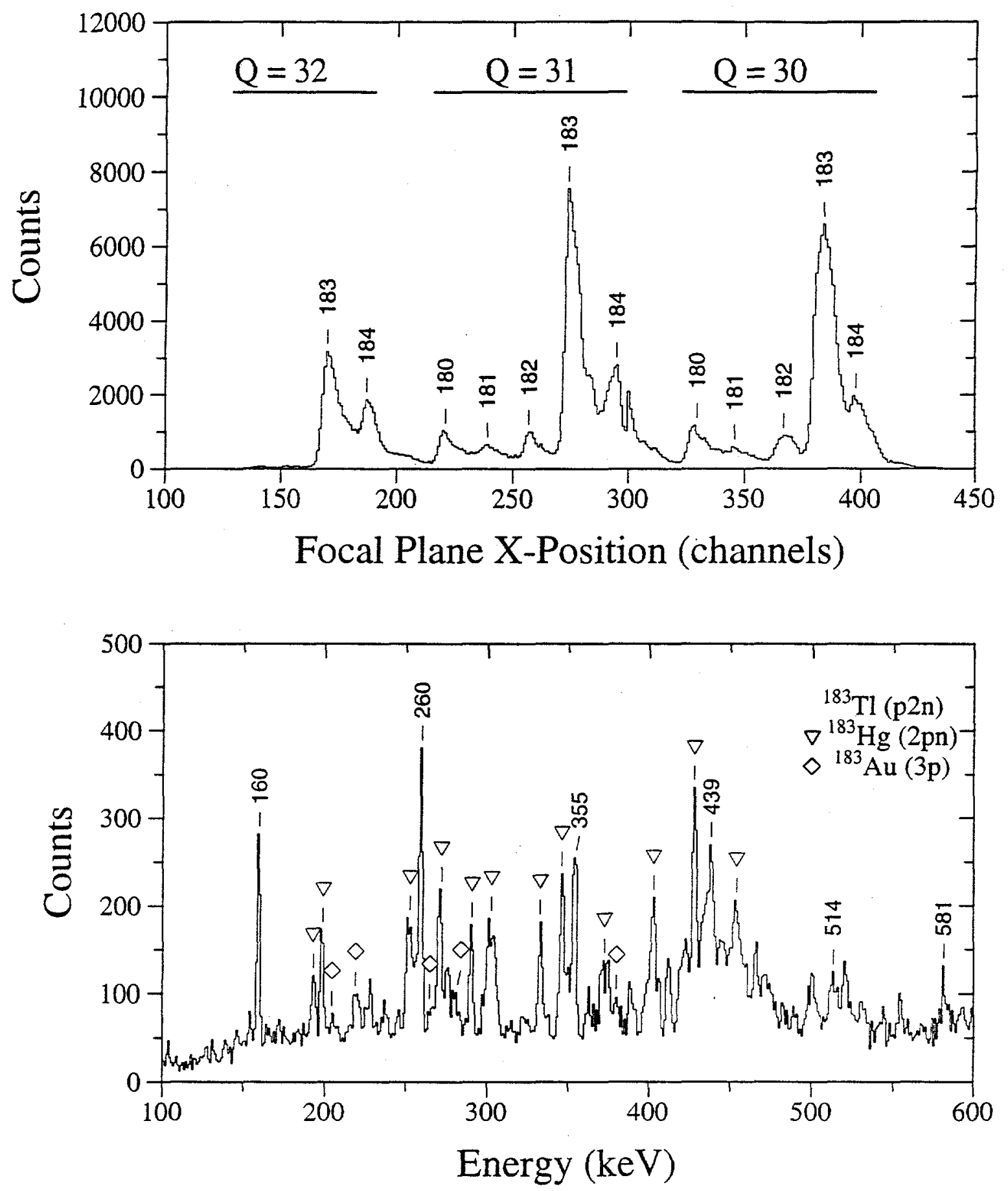

FIGURE 1. Top: $A / Q$ mass spectrum for ${ }^{78} \mathrm{Kr}$ on ${ }^{108} \mathrm{Pd}$ obtained at the horizontal position of the FMA focal plane. Peaks are labeled by the mass number, $A$, of the recoiling ions and fall into three groups corresponding to charge states $Q=30,31$, and 32. Bottom: Spectrum of prompt $\gamma$ rays from the target in coincidence with $A=183$ events in the focal plane $\mathrm{x}-\mathrm{y}$ position spectrum. Both spectra are generated under appropriate time-of-flight conditions (see text). 
${ }^{183} \mathrm{Tl}$

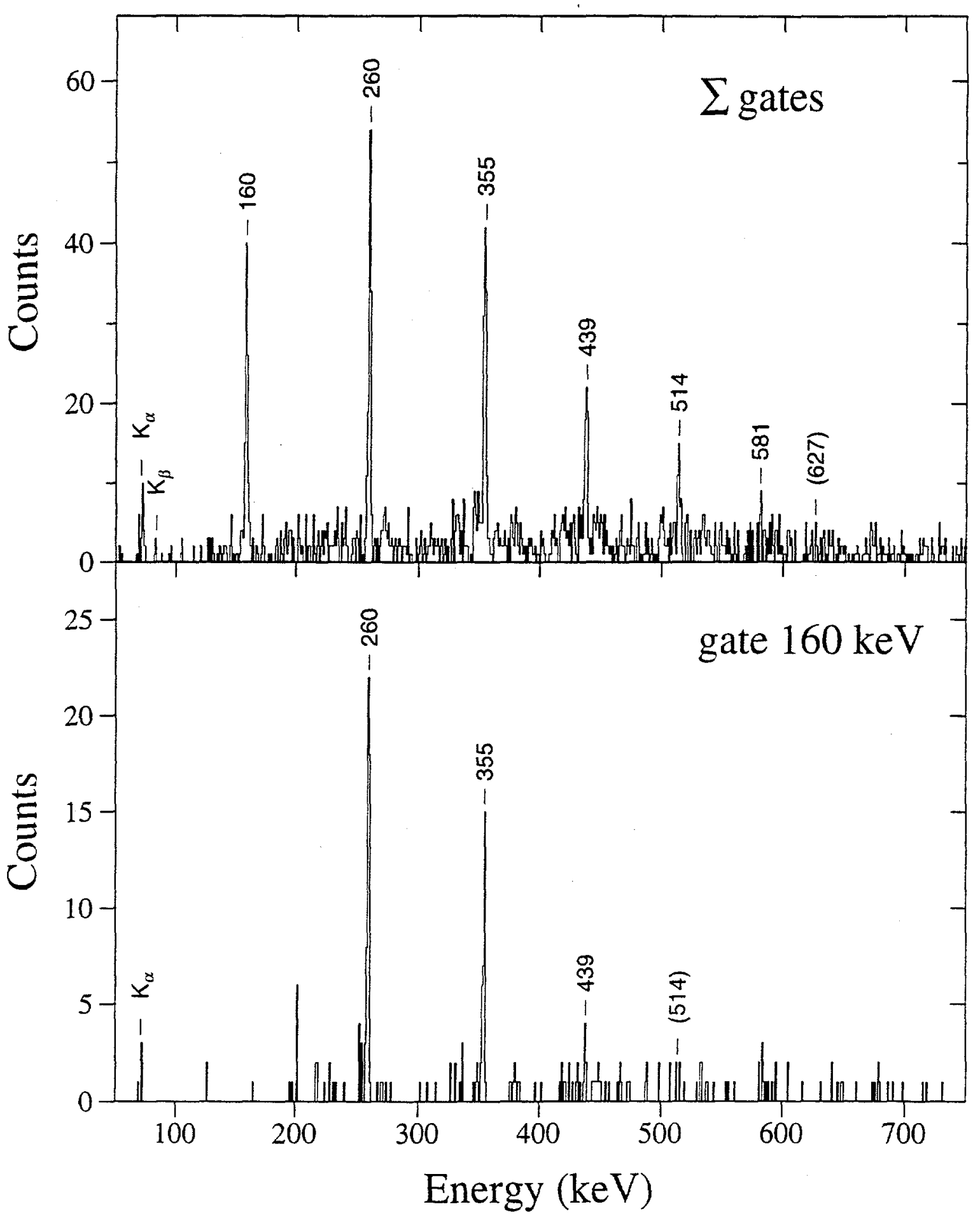

FIGURE 2. Sum of projections (top) from the mass 183 selected $E_{\gamma}-E_{\gamma}$ coincidence data with gates on the 160-, 260-, 355-, 439-, 514-keV lines and an "individual" gate (bottom) for comparison. Members of the rotational band (see text) are labeled by their energies in $\mathrm{keV}, \mathrm{x}$-rays of thallium are identified as well. 
Ref. [7], Fig. 8), one would expect to see in the gated $\gamma$-ray spectrum additional significant peaks at $400-450$ $\mathrm{keV}\left(11 / 2^{-} \rightarrow 9 / 2^{-}\right)$and probably at $100-150 \mathrm{keV}$. This is evidently not the case in the yrast spectrum of ${ }^{183} \mathrm{Tl}$, that is, there is no indication for strong linking transitions from the yrast sequence to the known [10] $9 / 2^{-}$isomeric state (oblate). By the same token, the yrast band in ${ }^{183} \mathrm{Tl}$ must be lower lying with respect to the $9 / 2^{-}$isomeric state than in the heavier isotopes.

The proposed level scheme for ${ }^{183} \mathrm{Tl}$ is shown in Fig. 3. Spins and parities of the levels above the $9 / 2^{-}$state are assigned by comparison with the yrast sequences in neighboring odd-mass $\mathrm{Tl}$ nuclei. The $160-\mathrm{keV} \gamma$ ray is identified as an E2 transition and placed at the bottom of the band based on intensity considerations. The inset of Fig. 3 shows the intensity after correction for detection efficiency and internal conversion as function of initial spin for the $\pi i_{13 / 2}$ bands in ${ }^{185,187} \mathrm{Tl}$ (labeled by the Nilsson quantum numbers [660]) [7] and the present data. A common normalization is used to ease the comparison of these intensity patterns. An E2 assignment for the strongly converted $160-\mathrm{keV} \gamma$ ray in ${ }^{183} \mathrm{Tl}$ ensures intensity conservation for the whole cascade, while $M 1$ or $E 1$ assignments are ruled cut for the same reason. It appears that the $\pi i_{13 / 2}$ band in ${ }^{183} \mathrm{Tl}$ is populated all the way down to the $13 / 2^{+}$bandhead due to its low excitation energy, even though the final decay out of this band is not clear yet.

How can the $\pi i_{13 / 2}$ band in ${ }^{183} \mathrm{Tl}$ decay? Depending on the excitation energy of the bandhead with respect to the $9 / 2^{-}$isomeric state, $E_{\text {rel }}$, there are two possible decay-out scenarios which define at the same time the upper and lower limit of $E_{\text {rei }}$. Upper limit: decay by M2 transition. Accepting the presence of a 11/2- level $421 \mathrm{keV}$ above the $9 / 2^{-}$state, a $13 / 2^{+} \rightarrow 9 / 2^{-} M 2$ transition would be competitive with a $13 / 2^{+} \rightarrow 11 / 2^{-} E 1$ transition if $E_{\gamma}(E 1) \leq 3 \mathrm{keV}$ according to a Weisskopf estimate $\left(E_{\text {rel }} \leq 424 \mathrm{keV}\right)$. In this scenario, the $E 1$ transition could not be observed. Lower limit: $\alpha$-decay to ${ }^{179} A u$. By knowing the location of the $13 / 2^{+}$level in the nucleus ${ }^{179} \mathrm{Au}\left(390 \mathrm{keV}\right.$ relative to the $9 / 2^{-}$level) [17] and the energy $E_{\alpha}\left(9 / 2^{-} \rightarrow 9 / 2^{-}\right)=6.38 \mathrm{MeV}$, reasonable predictions for the $\alpha$ energy of a $13 / 2^{+} \rightarrow 13 / 2^{+}$decay or the relative energy of the initial state can be made. The previous upper-limit estimate for the $13 / 2^{+}$state in ${ }^{183} \mathrm{Tl}$ and the estimate $\left(E_{\text {rel }}=410 \mathrm{keV}\right)$ obtained for an $6.40 \mathrm{MeV} \alpha$ decay from this state to the $13 / 2^{+}$level in the daughter nucleus ${ }^{179} \mathrm{Au}$ would be compatible. However, one would also expect competing $\gamma$ decay (of $M 2$ type) from this initial state to the $9 / 2^{-}$state in ${ }^{183} \mathrm{Tl}$ (estimated half-life $<100 \mathrm{~ns}$ ), which is not conclusive yet. For a lower-limit estimate of $E_{\text {rel }}$, it is assumed that a $0.3 \% \alpha$-decay branch could be observed and the following consideration is made. A decay to $13 / 2^{+}$level in the daughter nucleus ${ }^{179} \mathrm{Au}$ is calculated to be competitive with a $13 / 2^{+} \rightarrow 9 / 2^{-} M 2$ transition if $E_{\gamma}(M 2) \leq 95 \mathrm{keV}$ ( $E_{\text {rel }} \geq 95 \mathrm{keV}$ ). The energy of the corresponding $\alpha$ line is $E_{\alpha} \leq 6.10 \mathrm{MeV}$. So far, a search for a new $\alpha$-line in this energy range has lead to a negative result. However, it is possible that this line is embedded in a strong $E_{\alpha}=6.13 \mathrm{MeV}$ line from the decay of ${ }^{180} \mathrm{Hg}$, a contaminant reaction product in the present data. To proceed, an average value of the two limits, $E_{r e l}=256 \mathrm{keV}$, is assumed in the following discussion. Notice that a $256 \mathrm{keV} \mathrm{M2}$ transition with an estimated half-life of 800 ns could not be observed in prompt $\gamma$-ray spectroscopy.

Fig. 4 compares experimentally obtained prolate-oblate and oblate-oblate energy differences for the nearyrast coexisting level structures in $\mathrm{Tl}$ isotopes. These data are given relative to the $9 / 2^{-}$isomeric state (labeled [505]), where the prolate bandheads for ${ }^{185-189} \mathrm{Tl}$ have been derived from extrapolations of the levels observed at and above the $17 / 2^{+}$using a variable-moment-of-inertia fit [7]. The new information obtained for $\left.{ }^{183} \mathrm{~T}\right]$ is represented by the open symbols and bars (upper and lower limit for the relative energy of the prolate bandhead). For comparison, the energy differences between prolate bandheads (extrapolated $0_{2}^{+}$states) and the oblate deformed ground state in the $\mathrm{Hg}$ isotones are included as well. As stated above, these oblate states in $\mathrm{Hg}$ and $\mathrm{Tl}$ nuclei are viewed to be similarly deformed and thus can serve as a common "reference".

The oblate states in the $\mathrm{Tl}$ isotopes minimize in energy around $N=108$ and then slightly move up as function of neutron number, as can be seen in Fig. 4 by the systematic behavior of the $11 / 2^{-} \rightarrow 9 / 2^{-}$transitions (diamonds). For completeness, the $13 / 2_{1}^{+}$state (labeled [606]), found in the $\mathrm{Tl}$ isotopes for $A \geq 185$, is plotted as well. In ${ }^{185} \mathrm{Tl}$, this state interacts strongly with the $13 / 2^{+}$bandhead of the prolate structure (leading to a $\sim 30 \mathrm{keV}$ displacement). There is no indication yet for another $13 / 2^{+}$state in ${ }^{183} \mathrm{Tl}$ nor for a perturbation of the proposed $13 / 2^{+}$bandhead of the prolate structure (judging e.g. from a systematic comparison of the aligned angular momenta in the bands under discussion).

The prolate-oblate differences in $\mathrm{Tl}$ nuclei as a function of neutron number follow approximately the trend seen in the $\mathrm{Hg}$ isotones for $N \geq 104$ (dashed lines). However, near $N=103$ (neutron $i_{13 / 2}$ midshell) the prolate band in $\mathrm{Hg}$ nuclei reaches its minimum energy, while by the upper-limit estimate for ${ }^{183} \mathrm{Tl}$ the band in $\mathrm{Tl}$ nuclei is further downsloping with decreasing $N$. Interestingly, the ${ }^{179,181,183} \mathrm{Au}$ nuclei $[17,13]$ show a trend in prolate-prolate ([660]-[532]) differences (minimum at $N \leq 100$ ) that is perhaps comparable with $E_{\text {rel }}$ versus $N$ for the [660] bands in the $\mathrm{Tl}$ isotopes. On the other hand, for the even-mass $\mathrm{Pb}$ isotopes the trend 

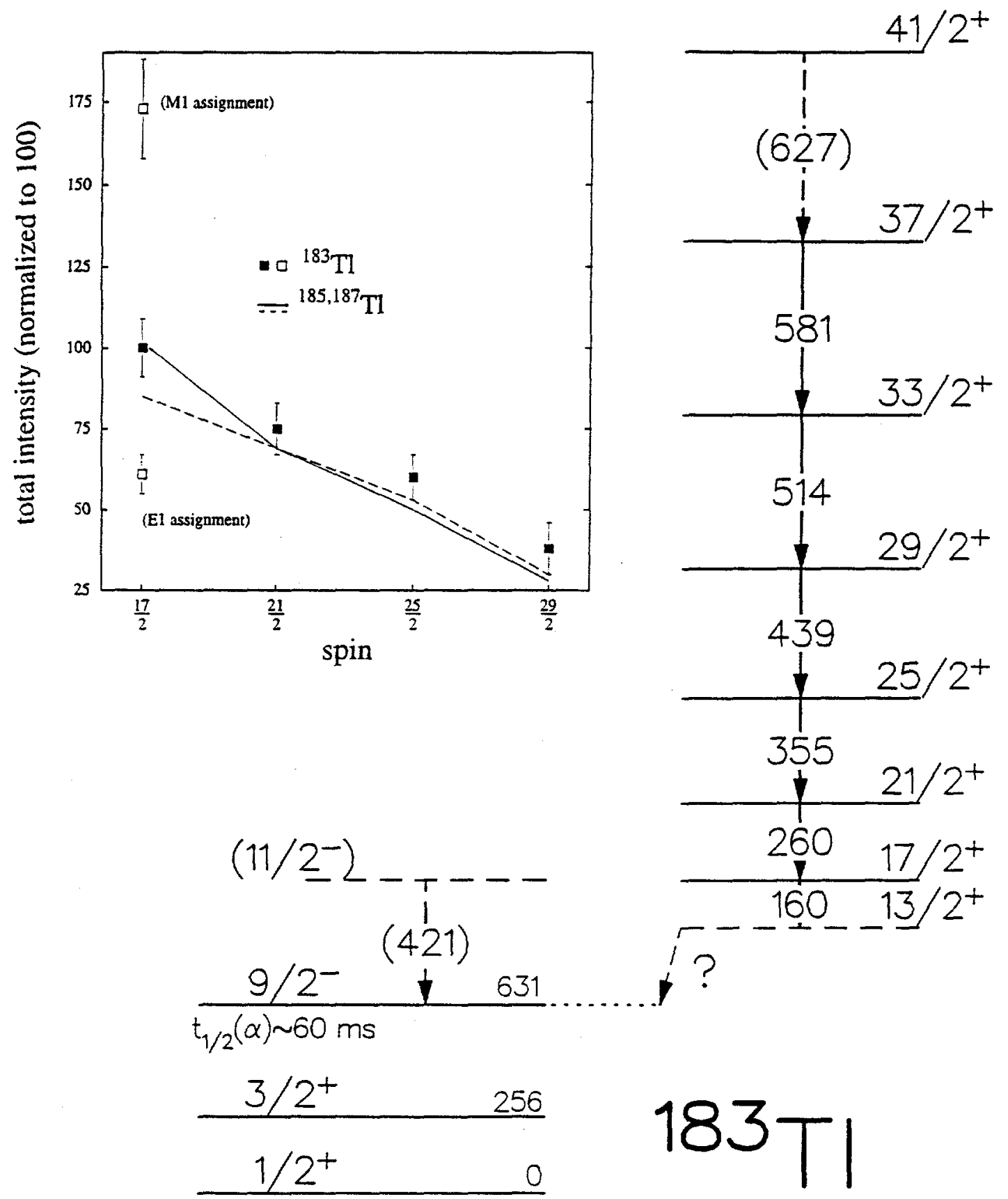


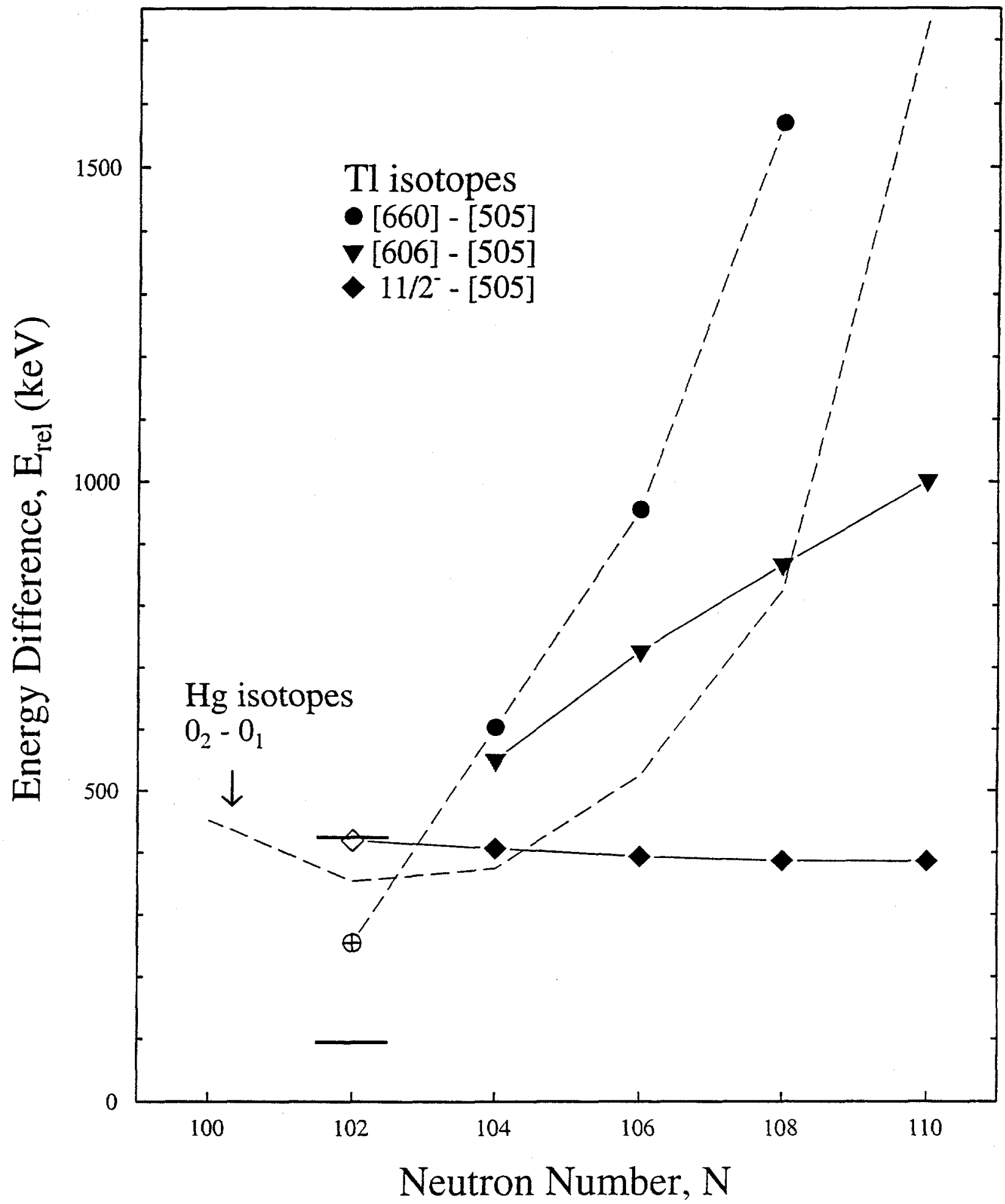

FIGURE 4. Energies of coexisting level structures in $\mathrm{Tl}$ isotopes relative to the corresponding [505]9/2- isomeric states versus neutron number as obtained from experiment. The data point at $N=102\left({ }^{183} \mathrm{Tl}\right)$ is an average of upper and lower limit estimates (see text). For comparison, the trend of the prolate-oblate energy difference in the Hg isotopes is indicated as well. 
of prolate-spherical $\left(\mathrm{O}_{3}^{+}-0_{1}^{+}\right)$energy differences found near $N=103$ [4] is similar to that for the $\mathrm{Hg}$ isotopes shown in Fig. 4. These findings indicate that the odd proton has considerable impact on the formation of the prolate minimum and gives rise to speculations such as polarization of the quadrupole core when coupling a deformation driving $i_{13 / 2}$ proton to it. Recent calculations [7] predict that the prolate states in odd-mass $\mathrm{Tl}$ nuclei continue to drop in energy past midshell, in agreement with the current results. However, as stated in Ref. [7], further theoretical investigations are necessary to better understand this behavior of the prolate states.

\section{CONCLUSIONS}

The yrast sequence in ${ }^{183} \mathrm{Tl}$ has been observed for the first time in recoil-mass and decay tagged $\gamma$-ray spectroscopic measurements, recently performed with GAMMASPHERE at the FMA and JUROSPHERE at RITU. While the level spacings of this new sequence resemble the well-deformed (prolate) excited bands in adjacent nuclei of $\mathrm{Hg}, \mathrm{Tl}$, and $\mathrm{Pb}$, its decay-out properties are different from those cases in two respects. (i) The rotational-like sequence is observed from medium spin down to the $I^{\pi}=13 / 2^{+}$bandhead. (ii) A strong $\gamma$-decay branch from the prolate band to a slightly-oblate structure, like in heavier Tl nuclei, is not observed. These features indicate that the prolate energy minimum in ${ }^{183} \mathrm{Tl}$ has dropped significantly compared to ${ }^{185} \mathrm{Tl}$ and minimizes below the neutron $i_{13 / 2}$ midshell, as predicted by theory. The low excitation energy of the band in ${ }^{183} \mathrm{Tl}$ possibly gives rise to a rare mode of $\alpha$-decay, from the $13 / 2^{+}$bandhead to a deformed $13 / 2^{+}$ state in the daughter nucleus ${ }^{179} \mathrm{Au}$, presently under investigation. These findings, made possible by recent instrumental developments, represent an extreme case of prolate-oblate shape competition.

This work was supported by the US Departmet of Energy under contracts nos. DE-FG05-87ER40361 (U. Tenn.), W-31-109-ENG-38 (ANL), DE-AC05-96OR22464 (ORNL), and in part by the US National Science Foundation.

\section{REFERENCES}

1. Wood J. L., et al.., Phys. Rep. 215, 211 (1992).

2. Heese J., et al., Phys. Lett. B 302, 390 (1993).

3. Baxter A. M., et al., Phys. Rev. C 48, R 2140 (1993).

4. Cocks J. F. C., et al., Europ. Phys. J. A 3, 17 (1998).

5. Kreiner, A. J., et al., Phys. Rev. C 38, 2674 (1988).

6. Reviol W., et al., Phys. Rev. C 49, R 587 (1994).

7. Lane G. J., et al., Nucl. Phys. A 586, 316 (1995).

8. Dracoulis G., et al., Phys. Lett. B 208, 365 (1988).

9. Paul E. S., et al., Phys. Rev. C 51, 78 (1995).

10. Batchelder, J. C., et al., Europ. Phys. J. A, submittted (1998).

11. Lee I. Y., Nucl. Phys. A 520,641c (1990).

12. Davids, C. N., et al., Nucl. Instrum. Methods Phys. Res. B 70, 358 (1992).

13. Mueller, W. F. et al., to be published (1998).

14. Table of Isotopes, Eighth Edition, Vol. II.

15. Jenkins, D., to be published.

16. Schrewe U. J. et al., Phys. Lett. B 91, 46 (1980).

17. Mueller, W. F., et al., Univ. of Tennessee Progress Report, March 1, 1997. 